Original Research Paper

\title{
Diabetic Retinopathy: Changes in Levels of Orosomucoids in Patients Supplemented with Beta Glucan and Vitamin D
}

\author{
${ }^{1}$ Richter Josef, ${ }^{2}$ Závorková Martina, ${ }^{1}$ Stiborova Ivana, ${ }^{1}$ Král Vlastimil and ${ }^{3}$ Vetvicka Vaclav \\ ${ }^{I}$ Zdravotní ústav se sídlem v Ústí nad Labem, Usti nad Labem, Czech Republic \\ ${ }^{2}$ Očni klinika UJEP Masarykova nemocnice, Krajská zdravotní, a.s., Usti nad Labem, Czech Republic \\ ${ }^{3}$ University of Louisville, Department of Pathology, Louisville, KY, USA
}

\author{
Article history \\ Received: 12-03-2019 \\ Revised: 27-03-2019 \\ Accepted: 13-04-2019 \\ Corresponding Author: \\ Vaclav Vetvicka \\ University of Louisville, \\ Department of Pathology, 511 \\ South Floyd Street, Louisville, \\ KY 40202, USA \\ Email: vaclav.vetvicka@louisville.edu
}

\begin{abstract}
The role of beta glucan in stimulating of various biological reactions is getting more and more attention. In this report, we focused on the effects of oral supplementation with beta glucan and vitamin D on changes in serum levels of orosomucoids in patients with diabetic retinopathy. We measured the level of orosomucoids and evaluated the effects of oral supplementation. In this study we report significant decrease of orosomucoids levels after the end of the three-month treatment. Our results support the fact that continual supplementation with glucan is necessary for diabetes melitus and diabetic retinopathy patients.
\end{abstract}

Keywords: Glucan, Diabetic Retinopathy, Vitamin D, Treatment, Orosomucoids

\section{Introduction}

Prevalence of Diabetes Mellitus (DM) shows steep incline, both in DM Type 1 (DM1) and less steeply in DM Type 2 (DM2) (Duh et al., 2017; Muhammad et al., 2017). Diabetic patients are endangered by various complications, influencing the quality of their life functions. Diabetic Retinopathy (DR) holds the most common complications of DM with current prevalence reaching more than 10 million wordwide (El-Beblawy et al., 2016). Regulation of immune functions and control of inflammatory processes are decisive in maintaining normal retinal functions. Chronic parainflammatory process leads to initiation and manifestation of several impairments, including DR (Nita et al., 2014). Factors directly related to prevention of DM and reduction of incidence are: (1) quality of lifestyle, (2) nutrition, (3) reduction of obesity, (4) related metabolic syndrome. Inducing production of several proteins, gradually occurring by weight increase, is dependent on fat cells. It is increased by oxidative stress leading to dysregulation of normal parainflammatory reaction and chronic inflammation (Duh et al., 2017; Hajer et al., 2008; Tsuboi et al., 2018). Recent studies monitoring relations between Orosomucoids (ORM) and pathogenesis of DM found that high levels of ORM are related to regulation of glucose levels. ORM, also known as $\alpha 1$-acid glycoprotein, is one of the acute-phase proteins exhibiting a variety of activities both in vitro and in vivo.

Risk of DM development increases with weight gain, together with an increase of clinical problems related to metabolic syndrome (Richter et al., 2018a; 2018b; Zhou et al., 2017). Authors emphasize that for evaluation of this relation, it is better to replace the BMI criterium by other measurements (such as WHR) (Zhou et al., 2017; Zavorkova et al., 2018). Incidence of additional comorbidities connected with high weight is also related to significant changes of nonspecific and specific immune responses. These changes involve, among others, Toll-like receptors that are present not only on immunocytes, but also on adipocytes and intestinal cells. They play a part in development of complex balance influencing normal immune system and metabolism (Wolowczuk et al., 2008). Fat cell dysfunction in obese population is one cause of DM2 development (Hajer et al., 2008). Weight reduction and increased physical activity are effective interventions leading to fat cell function improvement.

Several studies described positive correlation between fat weight and levels of proteins signaling inflammation (Engstrom et al., 2003). Weight reduction in obese individuals is closely correlated to decrease elevated levels of inflammation and inflammationsignaling proteins. Proinflammatory cytokines, 
produced by adipocytes and preadipocytes (such as TNF- $\alpha$ and IL-6) significantly influence production of these inflammatory proteins: (1) ORM, (2) fibrinogen, (3) alpha 1 antitrypsin, (4) haptoglobin, (5) ceruloplasmin CRP, (6) SAA. These proteins subsequently support the occurrence of numerous diseases (Jensen and Whitehead, 1998). Literature shows that persistent increased levels of acute inflammation proteins can predict significant increase in weight in middle age population. This relation can lead to the assumption about possible subsequent connection with metabolic syndrome, cardiovascular diseases, diabetes and other health problems (Muhammad et al., 2017; Engstrom et al., 2003; Jensen and Whitehead, 1998). Association of elevated levels of ORM, haptoglobin and CRP with an elevated risk of DM development was already described (Duh et al., 2017; Muhammad et al., 2017). This high risk of diabetes related to high levels of acute inflammatory protein can be accepted with one condition: additional possible mechanisms involved must be evaluated and described (Muhammad et al., 2017). High ORM levels on DM1 reflect endothelial dysfunction and subclinical atherosclerosis and can be considered as an early marker of renal injury (El-Beblawy et al., 2016). ORM can regulate food intake and homeostasis of energy (Sun et al., 2016). Modulation of ORM expression can be considered as a new approach in regulation of obesity and subsequent diseases related to metabolic syndrome (Cloetens et al., 2012). Immunomomodulatory mechanisms, drug transport, capillar quality maintenance and sphingolipid metabolism are mediated via ORM binding to CCR-5 or to beta chain of hemoglobin (Muhammad et al., 2017; Qin et al., 2016). Levels of ORM correlate with BMI, fat deposits, leptin levels and fasting glucose levels. This suggests that ORM can participate in regulation of energetic balance (Muhammad et al., 2017; Lei et al., 2016). These findings were further confirmed by using mice lacking ORM1. These animals clearly displayed aberrant energy homeostatis including increased body weight and fat mass (Sun et al., 2016).

ORM has several additional modulatory activities: (1) immunomodulation, (2) sphingolipid metabolism, (3) drug transport, (4) keeping of capillar barrier function (Luo et al., 2015). All these findings have resulted in current intensive research in evaluation of ORM functions, particularly in relation to physiology of energy. It is well known that physical stress leads to elevated levels of ORM and subsequent reduction of fatigue upon binding to $\mathrm{C}-\mathrm{C}$ chemokine receptor type 5 (Lei et al., 2016; Straczkowski et al., 2018). Together with additional proteins of inflammatory response, ORM is a good risk indicator of cardiovascular damage and cardiovascular disease (Lei et al., 2016). ORM is considered to be proof of association between obesity and periodontitis (Range et al., 2013). Interesting studies measuring ORM in urine of patients with DM1 might be used in induction risk evaluation with urinary tract problems concerning DR patients (Christiansen et al., 2010; Kustan et al., 2016; Talks et al., 2018). Lately, increased attention is focused on evaluation of ORM I and II subclasses. Fascinating studies describing increased ORM I expression in odontogenic myxoma and particularly regulatory abilities ORM II in hepatocellular carcinome (Garcia-Munoz et al., 2012) inspired the possibility to assess levels of ORM I and ORM II during diabetes. This is not only in relation of possible preventive actions, but also in monitoring of possible risk of complications known during DM.

Several studies demonstrated associations between levels of vitamin D and incerased risk of development of DM2 (Lu et al., 2018; Spiro and Buttriss, 2014). Vitamin D supplementation, resulting in changes of its levels, results in significant decrease of risk of DM2 (Zavorkova et al., 2018; Luo et al., 2015). Numerous studies found deficits in vitamin D levels not only in European countries, but worldwide (Tessari and Lante, 2017). Some of these countries already established a program of food fortification with vitamin D towards improved quality of health of entire populations ((Tessari and Lante, 2017). A complex preventive step aiming on reduction of risks of DM2 development is using supplementation with vitamin D, C and E (GarciaBailo et al., 2011). In addition, the importance of affecting inflammatory reaction as DM2 induction prevention, is proposed (Garcia-Bailo et al., 2011; Bashir and Choi, 2017).

Another possibility leading to weight reduction is influencing inflammatory response by addition of beta glucan, resulting in reduction of inflammatory response mediated by preadipocytes and adipocytes. The fact that a diet fortified with fiber containing high glucan content increased metabolic readines and can be used in DM2 patients is well established (Nita et al., 2014; McFarlin et al., 2013).

Some studies describe effects of food supplemented with glucan and psylicia on DM2 occurence (Aoe et al., 2017; McRae, 2018). Beta glucan is established to be an important part of nutrition, which is able to modulate dysregulation of metabolism connected with metabolic syndrome (El Khoury et al., 2012; Lyon and Reichert, 2010; Aoe et al., 2017). In addition, glucan can significantly decrease food consumption, regulate glycemic index of food and positively affect glucose metabolism in patients with DM2 (Straczkowski et al., 2018; Bashir and Choi, 2017; McRae, 2018). In our group of patients with DM2 and DR we demonstrated significant changes of lipid metabolism caused by improvements of vitamin D levels and after glucan application (Zavorkova et al., 2018). In 
the current study we follow-up the previous work and focus on evaluation of ORM levels after glucan and vitamin D supplementation.

\section{Materials and Methods}

\section{Protocol}

We explained the experimental protocol and obtained consent forms from all participating patients. This study was Institutional Review Board (Regional Masaryk University) approved and performed in full agreement with the Helsinki declaration (revised version 2000.09.01) and in full compliance with the Czech Republic's clinical testing rules.

\section{Patients}

Our initial group of patients consisted of 52 patients with Diabetic Retinopathy (DR) on one or both eyes. In addition, this disease was further complicated by Diabetic Macular Edema (DME). Initial criteria included Central Thickness of Retina (CTR) over $250 \mu \mathrm{m}$ and best corrective eye sharpness $4 / 6.3$ to $4 / 40$. Excluding criteria were classic laser photocoagulation of macula less then 3 months before the start of the study, previous intraocular treatment via anti-VEGF antibodies less then 6 months before the start of the study, additional macular disease and situation after pars plana vitrectomy. Average CTR at the start of the study was $448.2 \pm 132.3 \mu \mathrm{m}$ (median 441.5 ), average best corrective eye sharpness was $63.1 \pm 13.4$ (median 65 ) of letters.

A group of a total of 52 patients diagnosed with diabetic retinopathy was divided into three groups. Group A (20 patients) was supplemented with glucan and vitamin D, Group B (20 patients) was supplemented with vitamin D and placebo and Group C (12 patients) was a control group. In all patients we took blood at regular intervals for a 3 month period. All immunological and biochemical observations necessary to follow the development of disease, together with basic treatment using subthreshold micropulse laser of retina by an eye doctor was performed.

At the beginning of the study, the average BMI was $31.34 \pm 5.1$, ABSI levels $0.0856 \pm 0.00076$. Only 4 patients $(7.24 \%)$ had an optimal weight, 33 patients (60\%) has BMI over 30 and the rest had BMI over 32.5 $(32.76 \%)$. The starting level of vitamin $D$ was $14.19 \pm 5.8 \mathrm{ng} / \mathrm{mL}$. Three months of supplementation resulted in an increase to $22.45 \pm 7.46 \mathrm{ng} / \mathrm{ml}(\mathrm{p}=0,001)$, additional 3 months increased the vitamin $\mathrm{D}$ levels to $29.21 \pm 14.16 \mathrm{ng} / \mathrm{ml}$, which almost reached recommended levels. After six months, 3 patients had vitamin D levels below $10 \mathrm{ng} / \mathrm{ml}, 19$ patients had vitamin D levels over $30 \mathrm{ng} / \mathrm{ml}$.

\section{Glucan}

Yeast-derived insoluble Glucan \#300 (>85\% dry w/w basis) was purchased from Transfer Point (Columbia, SC, USA). This glucan contains $96 \%$ carbohydrates and $2.1 \%$ proteins. Neutral sugar analysis confirmed $91.3 \%$ glucose and $8 \%$ mannose. $500 \mathrm{mg}$ of glucan was taken on an empty stomach, followed by $100 \mathrm{~mL}$ of water and a 30-minute rest prior to any food intake. The interval of this food supplementation was 3 months. Placebo was identical in size, shape and color.

\section{Vitamin D}

Vitamin D (colecaciferol, D3) was manufactured by Merck (Darmstadt, Germany). One $\mathrm{ml}$ of solution contains 20,000 IU of vitamin D3, one drop contains 500 IU. All patients were instructed to ingest vitamin D with fat-containing food. The dose used in our experiments was based on weight, sex, age and phototype with $80 \%$ reduction during summer (May to September).

\section{Testing}

Serum levels of ORM were tested using diagnostic kits and controls manufactured by Siemens (Siemens Health Care Diagnostics, Newark, DE, USA). All measurements were performed immediately after blood collection.

\section{Statistical Analysis}

Paired $t$ test statistical significance was evaluated (GraphPad Prism 5.04; GraphPad Software, USA). An average and standard deviation was evaluated after determining standard value composition (D'Agostino, Pearson). In case of nonstandard composition, values were converted into logarithms.

\section{Results}

Our findings of levels of ORM levels in serum are shown in Fig. 1. Average levels at the beginning of the study were equal in all tested groups. Group A had an average level of ORM 97.6 $\pm 23.86 \mathrm{~g} / \mathrm{L}$, Group B $100.41 \pm 30.91 \mathrm{~g} / \mathrm{L}$ and Group C $99.45 \pm 24.8 \mathrm{~g} / \mathrm{L}$. Our findings at 3 and 6 months were different. In Group A we found significantly different values $(\mathrm{P}=0.001)$ at month 3. Ending glucan supplementation with subsequent vitamin D supplementation resulted in slow increase of ORM levels to original levels of $102.21 \pm 26.96 \mathrm{~g} / \mathrm{L}$ ( $\mathrm{p}=$ 0.0199). Our findings in Group B supplemented with vitamin $\mathrm{D}$ and placebo were indentical throughout the entire study $-100.41 \pm 30.91 \mathrm{~g} / \mathrm{L}$ at the beginning, $99.6 \pm 23.97$ $\mathrm{g} / \mathrm{L}$ after 3 months and $96.73 \pm 21.05 \mathrm{~g} / \mathrm{L}$ after 6 months. Similarly, levels of ORM in Group C were not changed $99.45 \pm 24.0 \mathrm{~g} / \mathrm{L}, \quad 95.9 \pm 24.0 \mathrm{~g} / \mathrm{L}$ and $95.0 \pm 25.0 \mathrm{~g} / \mathrm{L}$, respectively. Differences between levels found in Groups $\mathrm{B}$ and $\mathrm{C}$ were tested to be statistically insignificant. 

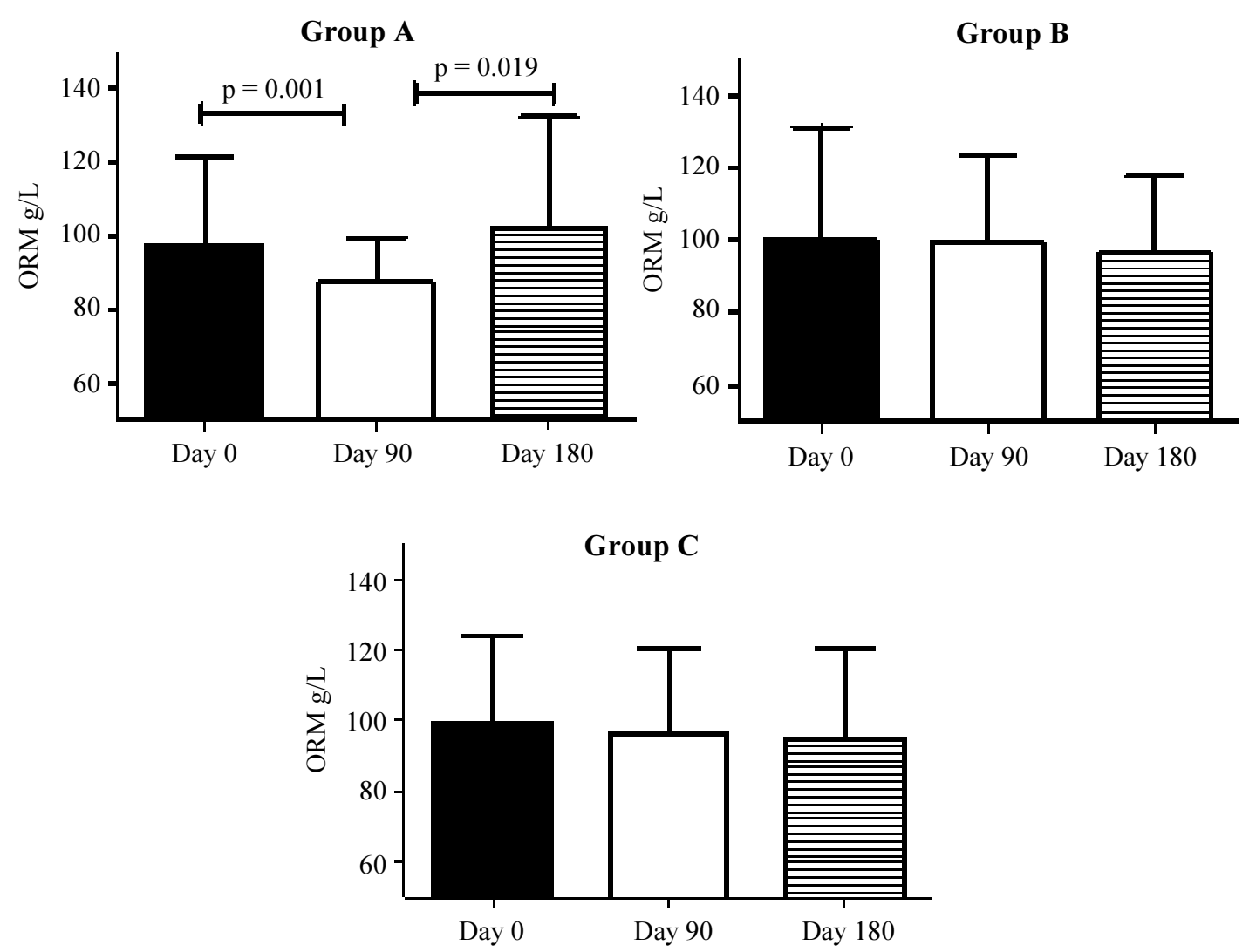

Fig. 1: Effects of glucan and vitamin D supplementation on levels of ORM. Group A - glucan and vitamin D; Group B - placebo and vitamin D; Group C - control. Average levels at the beginning of the study were equal in all tested groups. Our findings at 3 and 6 months were different. In Group A we found significantly different values at month 3. Ending glucan supplementation with subsequent vitamin D supplementation resulted in slow increase of ORM levels to original levels. Findings in Group B supplemented with vitamin D and placebo and in Group C were indentical throughout the entire study

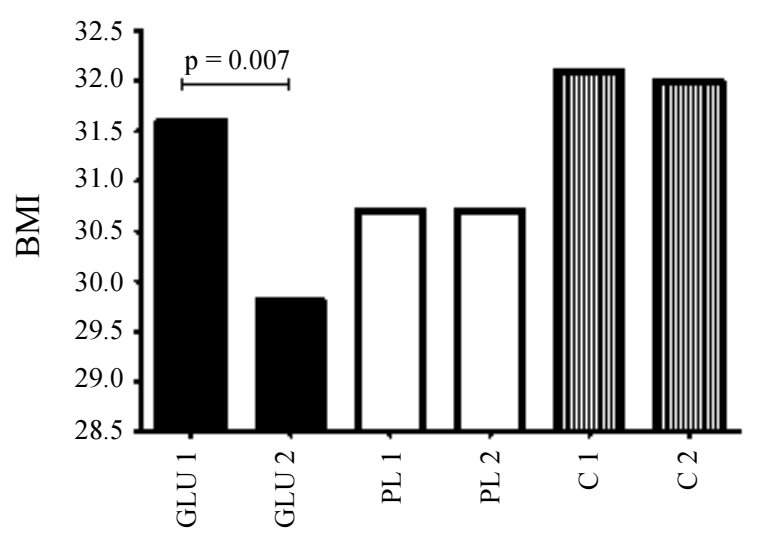

Fig. 2: BMI in tested groups. GLU - glucan and vitamin D (at the begining and the end of the study; PL - placebo and vitamin D (p $=0.9817)$; and $\mathrm{C}-$ vitamin $\mathrm{D}(\mathrm{p}=0.8448)$. Results represent mean $\pm \mathrm{SD}$. The results show significant decrease in weight in Group A (glucan and vitamin D), whereas in Group B (placebo and vitamin D) and Group C were seen only insignificant changes

Figure 2 focused on BMI in our groups. The results summarized in Fig. 2 show significant decrease in weight in Group A (glucan and vitamin D), whereas in
Group B (placebo and vitamin D) and Group C were seen only insignificant changes $(p=0.9817$ and $p=$ 0.8448 , resp.). 


\section{Discussion}

Inflammation and some mechanisms of immune response are two parts participating in development and progression of DR. It has been established that DM significantly affects neurovascular parts of retina in connection with vascular, neural, glial and immune mechanisms (Duh et al., 2017). Detailed elucidation of components mentioned above is based on attempts to find new therapeutic and preventive actions. These components are able to affect individual mechanisms playing a role in retina damage. Potential preventive regenerative steps might better control development of DR including treatment of early stages of the disease. In addition, they could help use better treatment based on individual conditions of each patient. Therefore, detailed evaluation of individual steps leading to developing DR is even more important. It seems elevated weight and particularly its steady increase, represent one of the important risk factors, resulting not only in DM, but also in DR. Significant decrease of glycol hemoglobin, increase of HDL levels and increase of blood pressure are common in obese individuals and represent clear risk factor for DR development (Zhou et al., 2017). Incremental increase of weight is accompanied by an increase of inflammatory proteins levels. Their levels are influenced by increasing numbers of adipocytes and preadipocytes producing numerous inflammatory proteins (Engstrom et al., 2003). A HDS-CC study showed connection among increasing levels of ORM, CRP, haptoglobin and risk of DM development (Muhammad et al., 2017). Inflammatory response results, among others, also in expression of serum amyloid A, is clearly connected with multifunctional apolipoproteins involved in metabolism of cholesterol and modulation of some immune mechanisms (Jensen and Whitehead, 1998). It is assumed that after physical stress, glucose levels are connected to ORM levels even in non-obese individuals. This potential relation is subject of several studies (Tsuboi et al., 2018). Evaluation of ORM levels in DM patients seems to be important most of all due to its wide spectrum of effects in case of DM development (Luo et al., 2015). ORM supports accumulation of glycogen in muscle cells and increases some cellular functions (Qin et al., 2016). ORM regulates food intake and energy homeostasis via leptin receptor signaling pathway (Sun et al., 2016). Based on these findings, one can hypothesize about possible regulation of obesity and/or metabolic problems of ORM expression regulation.

In our experimental group supplemented with glucan and vitamin D, we found significant increase in wellbeing and strong decrease of fatigue, which corresponds to the possibility of ORM modulation in similar conditions (Lei et al., 2016). ORM is considered to be an important indicator of an association between obesity and periodontitis (Range et al., 2013). Our experimental group could not confirm these associations, because our group of patients belong to a population with lower economics, education and health awareness. New findings describing two different members of ORM family, ORM1 and ORM2, offer new possibilities on how to better understand the part of ORM that plays in induction of DM and/or DR. ORM2 is considered to be an anti-inflammatory and immunodulatory agent with anti-neutrophil and anti-complement part, able to inhibit cytokine secretion. It addition, it is involved in regulation of angiogenesis and in regulation of capillary permeability (Duh et al., 2017; Hajer et al., 2008; Fang et al., 2015). ORM1 is assumed to play a role in induction of cancer development and is involved in regulation of angiogenesis (Muhammad et al., 2017). We based our study of ORM in DM2 on published findings of relation between ORM and DM1 (ElBeblawy et al., 2016). Our data showed that evaluation of serum levels of ORM and its dynamics related to DM complication is valuable. In addition, we also tried to measure ORM in urine (unpublished results). Our study showed the evaluation of ORM in urine of patients with DM and DR might be important, which agrees with results of other laboratories (Christiansen et al., 2010; KUstan et al., 2016; Talks et al., 2018). In all of our patients, we found significant increase of ORM levels when compared to normal population of the same age. Elevated levels of ORM in patient urine with DR might reflect not only risk of urinary tract infection (which could be eliminated by bacteriological evaluation), but another possible complication: risk of damaged glomerulal functions.

In the last decade, Europe and probably the whole world, observed a significant deficit of vitamin D in the entire population (Spiro and Buttriss, 2014). Steadily increasing knowledge about the role of vitamin $\mathrm{D}$ deficit in appearance and development of clinical progress of several diseases results in increased interests in this subject; not only from the medical and scientific profession, but also from the general population. Attempts to increase the interests of general public in consumption of food with higher content of vitamin D (either naturally or as a supplement) is increasing (Spiro and Buttriss, 2014; Tessari and Lante, 2017; Aoe et al., 2017). Lately, an association between vitamin D deficit and risk of DM2 development is being discussed in the literature ( $\mathrm{Lu}$ et al., 2018). Our findings show that vitamin D supplementation helps to improve several clinical manifestation of DM2 and its complications (Zavorkova et al., 2018; Christiansen et al., 2010). Risk of DR development in overweight or obese individuals is well documented (Zhou et al., 2017) and further confirmed by our findings. Our group of DR patients revealed that starting levels of vitamin D are approximately $20 \%$ lower when compared with patients with DM2. In addition, even 6 month supplementation did not cause an increase upto normal levels. Dynamics of vitamin D 
levels in our group was $14.2 \pm 5.9 \mathrm{ng} / \mathrm{ml}$ at start, $22.5 \pm 7.5 \mathrm{ng} / \mathrm{ml}$ after three months and $29.2 \pm 14.2 \mathrm{ng} / \mathrm{ml}$ after six months. The recomended value $(30 \mathrm{ng} / \mathrm{ml})$ was reached only in $36.5 \%$ of patients. Based on detailed evaluation, we concluded that the reason was inadequate adherence to recommended conditions of vitamin D supplementation.

Effects of glucan in prevention and treatment of obesity and clinical manifestation of DM2 were tested both in experimental and clinical studies (Hajer et al., 2008; Richter et al., 2018a; 2018b; Lu et al., 2018). Clinical trials confirmed that glucan supplementation resulted in a decrease of glycemic index and lipid metabolism, particularly cholesterol levels (Lu et al., 2018; Sima et al., 2018). Findings of changes in visceral obesity after glucan treatment, accompanied with decrease of weight and BMI are important (Wang et al., 2016). The positive effects of glucan in prevention and treatment of metabolic syndrome resulted in food fortification with glucan (Cloetens et al., 2012; McRae, 2018; El Khoury et al., 2012). It seems that food fortification with high fiber content has positive effects in regulation of metabolism. This can be used in prevention and management of DM (Richter et al., 2018a; 2018b). In addition, we found significant BMI decrease after supplementation with glucan and vitamin D. As the groups with vitamin D, but without glucan, showed no differences between start and end values, it is safe to assume that glucan is the supplement responsible for this change. Another important effect of glucan are improvements of salivary immunity (McFarlin et al., 2013) and gastrointestinal tract microbiom with positive effects on risk factors of cardiovascular diseases (Fang et al., 2015).

\section{Conclusion}

This study is a follow-up of previous observation showing effects of glucan on lipid metabolism and obesity in patients with DAR (Richter et al., 2018a; 2018b; Zavorkova et al., 2018). In this study we report significant decrease of ORM levels after the end of the three-month treatment. Our results support the fact that continual supplementation with glucan is necessary for DM and DR patients. We understand that in a population with poor dietary habits, this might be difficult. A direct addition of glucan and fiber into some food could represent a solution to this problem.

\section{Acknowledgment}

The authors are thankfull to Ms. Tracey Bender for her editorial help.

\section{Author's Contributions}

All authors equally contributed to this study.

\section{Ethics}

This study was Institutional Review Board (Regional Masaryk University) approved and performed in full agreement with the Helsinki declaration.

\section{References}

Aoe, S., Y. Ichinose, N. Kohyama, K. Komae and A. Takahashi et al., 2017. Effects of high beta-glucan barley on visceral fat obesity in Japanese individuals: A randomized, double-blind study. Nutrition, 42: 1-6. DOI: 10.1016/j.nut.2017.05.002

Bashir, K.M.I. and J.S. Choi, 2017. Clinical and physiological perspectives of beta-glucans: The past, present and future. Int. J. Mol. Sci., 18: 1906-1906. DOI: $10.3390 /$ ijms 18091906

Cloetens, L., M. Ulmius, A. Johansson-Persson, B. Åkesson and G. Önning, 2012. Role of dietary betaglucans in the prevention of the metabolic syndrome. Nutr. Rev., 70: 444-458.

DOI: $10.1111 /$ j.1753-4887.2012.00494.x

Christiansen, M.S., E. Hommel, L. Friberg, J. Mølvig and E. Magid et al., 2010. Increased urinary orosomucoid excretion is not related to impaired renal function in patients with type 2 diabetes. J. Diabetes Complicat., 24: 28-36.

DOI: $10.1016 /$ j.jdiacomp.2008.08.001

Duh, E.J., J.K. Sun and A.W. Stitt, 2017. Diabetic retinopathy: Current understanding, mechanisms and treatment strategies. JCI Insight. 2: e93751-e93751. DOI: $10.1172 /$ jci.insight.93751

El-Beblawy, N.M., N.G. Andrawes, E.A. Ismail, B. ElSaid Enany and H.S.A. El-Seoud et al., 2016. Serum and urinary orosomucoid in young patients with type 1 diabetes: a link between inflammation, microvascular complications and subclinical atherosclerosis. Clin. Appl. Thromb. Hemost., 22: 718-726. DOI: 10.1177/1076029616637185

El Khoury, D., C. Cuda, B.L. Luhovyy and G.H. Anderson, 2012. Beta glucan: Health benefits in obesity and metabolic syndrome. J. Nutr. Metab. DOI: $10.1155 / 2012 / 851362$

Engstrom, G., B. Hedblad, L. Stavenow, P. Lind and L. Janzon et al. 2003. Inflammation-sensitive plasma proteins are associated with future weight gain. Diabetes, 52: 2097-2101.

DOI: $10.2337 /$ diabetes.52.8.2097

Fang, T., M. Cui, J. Sun, C. Ge and F. Zhao et al., 2015. Orosomucoid 2 inhibits tumor metastasis and is upregulated by CCAAT/enhancer binding protein beta in hepatocellular carcinomas. Oncotarget, 6: 16106-61619. DOI: 10.18632/oncotarget.3867 
Garcia-Bailo, B., A. El-Sohemy, P.S. Haddad, P. Arora and F. BenZaied et al., 2011. Vitamins D, C and E in the prevention of type 2 diabetes mellitus: modulation of inflammation and oxidative stress. Biologics, 5: 7-19. DOI: 10.2147/BTT.S14417

Garcia-Munoz, A., M.A. Rodriguez, R. Bologna-Molina, F.E. Cázares-Raga and F.C. Hernández-Hernández et al., 2012. The orosomucoid 1 protein (alphal acid glycoprotein) is overexpressed in odontogenic myxoma. Proteome Sci., 10: 49-49. DOI: $10.1186 / 1477-5956-10-49$

Hajer, G.R., T.W. van Haeften and F.L. Visseren, 2008. Adipose tissue dysfunction in obesity, diabetes and vascular diseases. Eur. Heart J., 29: 2959-2971. DOI: 10.1093/eurheartj/ehn387

Jensen, L.E. and A.S. Whitehead, 1998. Regulation of serum amyloid a protein expression during the acute-phase response. Biochem. J., 334: 489-503. DOI: $10.1042 /$ bj3340489

Kustan, P., B. Szirmay, Z. Horvath-Szalai, A. Ludány and Á. Lakatos et al., 2016. Urinary orosomucoid: Validation of an automated immune turbidimetric test and its possible clinical use. Biochem. Med. (Zagreb), 26: 421-430.

DOI: 10.11613/BM.2016.044

Lei, H., Y. Sun, Z. Luo, G. Yourek and H. Gui et al., 2016. Fatigue-induced orosomucoid 1 acts on C-C chemokine receptor type 5 to enhance muscle endurance. Sci. Rep., 6: 18839-18839. DOI: $10.1038 /$ srep 18839

Lu, L., D.A. Bennett, I.Y. Millwood, S. Parish and M.I. McCarthy et al., 2018. Association of vitamin D with risk of type 2 diabetes: A Mendelian randomisation study in European and Chinese adults. PLoS Med., 15: e1002566-e1002566.

DOI: 10.1371/journal.pmed.1002566

Luo, Z., H. Lei, Y. Sun, X. Liu and D.F. Su, 2015. Orosomucoid, an acute response protein with multiple modulating activities. J. Physiol. Biochem., 71: 329-340. 10.1007/s13105-015-0389-9

Lyon, M.R. and R. Reichert, 2010. The effect of a novel viscous polysaccharide along with lifestyle changes on short-term weight loss and associated risk factors in overweight and obese adults: An observational retrospective clinical program analysis. Altern. Med. Rev., 15: 68-75. PMID: 20359270

McFarlin, B.K., K.C. Carpenter, T. Davidson and M.A. McFarlin, 2013. Baker's yeast beta glucan supplementation increases salivary $\operatorname{IgA}$ and decreases cold/flu symptomatic days after intense exercise. J. Diet. Suppl., 10: 171-183. DOI: $10.3109 / 19390211.2013 .820248$

McRae, M.P., 2018. Dietary fiber intake and type 2 diabetes mellitus: An umbrella review of metaanalyses. J. Chiropr. Med., 17: 44-53.

DOI: $10.1016 / \mathrm{j} . j \mathrm{~cm} .2017 .11 .002$
Muhammad, I.F., Y. Borne, G. Ostling, C. Kennbäck and M. Gottsäter et al., 2017. Acute phase proteins as prospective risk markers for arterial stiffness: The Malmo Diet and Cancer cohort. PLoS One, 12: e0181718-e0181718.

DOI: 10.1371/journal.pone.0181718

Nita, M., A. Grzybowski and F.J. Ascaso, 2014. Agerelated macular degeneration in the aspect of chronic low-grade inflammation (pathophysiological parainflammation). Mediators Inflamm., 2014: 930671-930671. DOI: 10.1155/2014/930671

Qin, Z., J.J. Wan, Y. Sun, P.Y. Wang and D.F. Su et al., 2016. ORM Promotes skeletal muscle glycogen accumulation via CCR5-activated AMPK pathway in mice. Front. Pharmacol. DOI: $10.3389 /$ fphar.2016.00302

Range, H., C. Poitou, A. Boillot, C. Ciangura and S. Katsahian et al., 2013. Orosomucoid, a new biomarker in the association between obesity and periodontitis. PLoS One, 8: e57645-e57645. DOI: 10.1371/journal.pone.0057645

Richter, J., M. Zavorkova, V. Vetvicka, I. Liehneová and V. Kral et al., 2018a. Effects of $\beta$-glucan and vitamin D supplementation on inflammatory parameters in patients with diabetic retinopathy. J. Diet. Suppl. DOI: 10.1080/19390211.2018.1458769

Richter, J., M. Závorková, V. Vetvicka, I. Liehneová and V. Král, et al., 2018b. Vitamin D and $\beta$-glucan supplementation affects levels of leptin, apolipoproteins and general nutrition state in patients with diabetic retinopathy. Edorium J. Pathol. DOI: 10.5348/100009P03RJ2018OA

Sima, P., L. Vannucci and V. Vetvicka, 2018. $\beta$-Glucans and cholesterol (Review). Int. J. Mol. Med., 41: 1799-1808. DOI: $10.3892 /$ ijmm.2018.3411

Spiro, A. and J.L. Buttriss, 2014. Vitamin D: An overview of vitamin D status and intake in Europe. Nutr. Bull., 39: 322-350. DOI: 10.1111/nbu.12108

Straczkowski, M., A. Nikolajuk, R. Majewski, R. Filarski and M. Stefanowicz et al., 2018. The effect of moderate weight loss, with or without $(1,3)(1,6)$ beta-glucan addition, on subcutaneous adipose tissue inflammatory gene expression in young subjects with uncomplicated obesity. Endocrine, 61: 275284. DOI: $10.1007 / \mathrm{s} 12020-018-1619-\mathrm{z}$

Sun, Y., Y. Yang, Z. Qin, J. Cai and X. Guo et al., 2016. The acute-phase protein orosomucoid regulates food intake and energy homeostasis via leptin receptor signaling pathway. Diabetes, 65: 1630-1641. DOI: $10.2337 / \mathrm{db} 15-1193$

Talks, B.J., S.B. Bradwell, J. Delamere, W. Rayner and A. Clarke et al., 2018. Urinary alpha-1-acid glycoprotein is a sensitive marker of glomerular protein leakage at altitude. High Alt. Med. Biol., 19: 295-298. DOI: 10.1089/ham.2018.0017 
Tessari, P. and A. Lante, 2017. A multifunctional bread rich in beta glucans and low in starch improves metabolic control in type 2 diabetes: A controlled trial. Nutrients, 9: 297-297. DOI: $10.3390 /$ nu9030297

Tsuboi, A., S. Minato, M. Yano, M. Takeuchi and K. Kitaoka et al., 2018. Association of serum orosomucoid with 30-min plasma glucose and glucose excursion during oral glucose tolerance tests in non-obese young Japanese women. BMJ Open Diabetes Res. Care, 6: e000508-e000508. DOI: $10.1136 /$ bmjdrc-2018-000508

Wang, Y., N.P. Ames, H.M. Tun, S.M. Tosh and P.J. Jones et al., 2016. High molecular weight barley $\beta$ glucan alters gut microbiota toward reduced cardiovascular disease risk. Front. Microbiol., 7: 129-129. DOI: 10.3389/fmicb.2016.00129
Wolowczuk, I., C. Verwaerde, O. Viltart, A. Delanoye and M. Delacre et al., 2008. Feeding our immune system: Impact on metabolism. Clin. Dev. Immunol., 2008: 639803-639803.

DOI: $10.1155 / 2008 / 639803$

Zavorkova, M., V. Vetvicka, J. Richter, V. Kral and I. Liehnova et al., 2018. Effects of glucan and vitamin D supplementation on obesity and lipid metabolism in diabetic retinopathy. Open Biochem. J., 12: 36-45.

DOI: $10.2174 / 1874091 \mathrm{X} 01812010036$

Zhou, Y., Y. Zhang, K. Shi and C. Wang, 2017. Body mass index and risk of diabetic retinopathy: A metaanalysis and systematic review. Medicine (Baltimore), 96: e6754-e6754.

DOI: 10.1097/MD.0000000000006754 